TITLE:

\title{
Measured total cross sections of slow neutrons scattered by gaseous and liquid $\mathrm{H}-2(2)$
}

\section{$\operatorname{AUTHOR}(\mathrm{S}):$}

Atchison, F; van den Brandt, B; Brys, T; Daum, M; Fierlinger, P; Hautle, P; Henneck, R; ... Zmeskal, J; Hino, M; Utsuro, $M$

\section{CITATION:}

Atchison, F ... [et al]. Measured total cross sections of slow neutrons scattered by gaseous and liquid H-2(2). Physical Review Letters 2005, 94(21): 212502.

\section{ISSUE DATE:}

2005-06-03

URL:

http://hdl.handle.net/2433/39925

RIGHT:

Copyright 2005 American Physical Society 


\title{
Measured Total Cross Sections of Slow Neutrons Scattered by Gaseous and Liquid ${ }^{2} \mathrm{H}_{2}$
}

\author{
F. Atchison, B. van den Brandt, T. Bryś, ${ }^{*}$ M. Daum, P. Fierlinger, ${ }^{\dagger}$ P. Hautle, R. Henneck, K. Kirch ${ }^{\ddagger}$ J. Kohlbrecher, \\ G. Kühne, J. A. Konter, A. Pichlmaier, and A. Wokaun* \\ Paul Scherrer Institut (PSI), CH-5232 Villigen PSI, Switzerland ${ }^{\S}$ \\ K. Bodek, M. Kasprzak," and M. Kuźniak" \\ Institute of Physics, Jagiellonian University, Cracow, Poland \\ P. Geltenbort \\ Institut Laue-Langevin (ILL), Grenoble, France \\ M. Giersch and J. Zmeskal \\ Institute for Medium Energy Physics, Austrian Academy of Sciences, Vienna, Austria \\ M. Hino and M. Utsuro ${ }^{\text {I }}$ \\ Kyoto University, Kyoto, Japan \\ (Received 22 November 2004; published 3 June 2005)
}

\begin{abstract}
The total scattering cross sections for slow neutrons with energies $E$ in the range $300 \mathrm{neV}$ to $3 \mathrm{meV}$ for gaseous and liquid ortho- ${ }^{2} \mathrm{H}_{2}$ have been measured. The cross sections for ${ }^{2} \mathrm{H}_{2}$ gas are found to be in excellent agreement with both the Hamermesh and Schwinger and the Young and Koppel models. For liquid ${ }^{2} \mathrm{H}_{2}$, we confirm the existing experimental data in the cold neutron range and the discrepancy with the gas models. We find a clear $1 / \sqrt{E^{\prime}}$ dependence at low energies for both states. A simple explanation for the liquid ${ }^{2} \mathrm{H}_{2}$ cross section is offered.
\end{abstract}

DOI: 10.1103/PhysRevLett.94.212502

The first complete treatment of thermal neutron scattering by the hydrogen molecule was made by Schwinger and Teller [1] in connection with the experimental determination of the range and spin dependence of the neutronproton interaction. This work was extended by Hamermesh and Schwinger (HS) [2,3] to the deuterium molecule so that similar information could be obtained about the neutron-deuteron interaction. Growing interest in the study of molecular systems and in neutron scattering led to the development of the general theoretical treatment of neutron scattering by molecules by Zemach and Glauber [4]. Based on that, Young and Koppel (YK) [5,6] gave a first fully detailed treatment of the hydrogen molecule valid for (i) neutrons with energies in the range from the Debye temperature (about $7 \mathrm{meV}$ ) to a few eV, (ii) both ${ }^{1} \mathrm{H}_{2}$ and ${ }^{2} \mathrm{H}_{2}$, and (iii) the gaseous and (with caution) liquid states; good agreement with the experimental data for ${ }^{1} \mathrm{H}_{2}$ available at that time [7] was obtained, including cross section values in the energy region below $7 \mathrm{meV}$.

A specific interest in the scattering of neutrons by hydrogen comes from work to develop cold neutron sources; experimental measurements for liquid and solid ${ }^{1} \mathrm{H}_{2}$ and ${ }^{2} \mathrm{H}_{2}$ [8,9] exposed differences between the values for cross sections measured and those calculated using the YK model, particularly for neutrons below $4 \mathrm{meV}$ interacting with ${ }^{2} \mathrm{H}_{2}$ liquid. Several explanations and models [10-15] have been put forward to explain these discrepancies.

Our interest is the use of solid ${ }^{2} \mathrm{H}_{2}$ for the production of ultracold neutrons (UCN) $[16,17]$. We are currently en-
PACS numbers: 28.20.Cz, 25.40.Dn, 61.12.Ex, 61.25.Em

gaged in an experimental program to measure its neutron scattering properties extending earlier studies [18-20]. However, given the somewhat uncertain situation regarding scattering from the liquid and the rather small amount of experimental data available [21], we have included experiments to measure the cross sections for the scattering of cold and very cold neutrons ( $\mathrm{CN}$ and $\mathrm{VCN}$, respectively) by gaseous and liquid ${ }^{2} \mathrm{H}_{2}$. This Letter presents the measured cross sections for ${ }^{2} \mathrm{H}_{2}$ gas and liquid and also compares the results with theory to show where discrepancies arise.

The measurements have been performed using cold neutrons at the SANS-I instrument [22] of the SINQ facility at PSI and with VCN and UCN at the PF-2 instrument [23] of the Institute Laue-Langevin (ILL). The total cross sections have been measured using the transmission technique

$$
\sigma=\frac{\ln \left(I_{0} / I\right)}{\rho d},
$$

where $I_{0}$ and $I$ are the transmitted intensities for the empty and full sample cells, $\rho$ is the density, and $d$ is the thickness of the sample cell. Neutron energy $(E)$ measurements have been made using velocity selection and time of flight and converted to in-medium kinetic energy $\left(E^{\prime}=E-U\right.$; see, e.g., Ref. [24]), using a Fermi pseudopotential, $U$, of $88 \mathrm{neV}$ for liquid deuterium [25]. Because of its low density, refraction can be neglected in the case of gaseous deuterium (i.e., $U \approx 0$ and $E^{\prime} \approx E$ even for $\mathrm{UCN}$ ). The 
experimental setups, data treatment procedures, and the solid ${ }^{2} \mathrm{H}_{2}$ cross section measurements are described in detail elsewhere [26,27]. The cryogenic target cell for the ${ }^{2} \mathrm{H}_{2}$ is the same as used earlier [28].

The ${ }^{2} \mathrm{H}_{2}$ had a purity of at least $99.95 \%$ and was prepared with a high ortho concentration of $c_{o}=(98.6 \pm$ $0.4) \%$ [28]. The para- ${ }^{2} \mathrm{H}_{2}$ fraction contributes about $1 / v \times$ $\left(1-c_{o}\right) \times 220 \mathrm{~b} \cdot \mathrm{m} / \mathrm{s}$ to the measured cross sections (estimated using [29]). The maximum possible $\mathrm{H}$ contamination $c_{\mathrm{H}}=0.0005$ contributes $c_{\mathrm{H}} \times 82 \mathrm{~b}$ incoherent scattering and $1 / v \times c_{\mathrm{H}} \times 730 \mathrm{~b} \cdot \mathrm{m} / \mathrm{s}$ absorption cross section. These contributions are comparable to the absorption on deuterons $(1 / v \times 1.1 \mathrm{~b} \cdot \mathrm{m} / \mathrm{s})$ and negligible (see measured cross sections below).

The measurements with ${ }^{2} \mathrm{H}_{2}$ gas have been performed at a temperature of $(25.0 \pm 0.3) \mathrm{K}$ with the target connected to a (warm) gas tank at a pressure of $(1.09 \pm 0.02) \times$ $10^{5} \mathrm{~Pa}$. The gas density in the target cell is calculated (using parameters from [30]) to be $\rho_{\text {gas }}^{-1}=(1.63 \pm$ 0.04) $\mathrm{dm}^{3} /$ mol. The measurements with liquid ${ }^{2} \mathrm{H}_{2}$ have been performed at $(19.1 \pm 0.1) \mathrm{K}$. The liquid ${ }^{2} \mathrm{H}_{2}$ density $\rho_{\text {liq }}^{-1}=(23.3 \pm 0.1) \mathrm{cm}^{3} / \mathrm{mol}$ was taken from [31]. Solid angle corrections are small and range between $10^{-2}$ and $10^{-3}$. A major experimental uncertainty is the actual target thickness $d$, especially with thin target windows, which bulge. We used windows machined from aluminum alloy $\left(\mathrm{AlMg}_{3} / \mathrm{AA5754}\right)$ rods to thicknesses of $150-200 \mu \mathrm{m}$. The windows must withstand typically a pressure of $1 \mathrm{~atm}$ against vacuum. The nominal target thickness is $d=$ $10 \mathrm{~mm}$ for flat windows. In the analysis effective target thicknesses were used, $d=10 \mathrm{~mm}$ for the $\mathrm{CN}$ and VCN data for one set of windows and $d=11.1 \mathrm{~mm}$ for the UCN data taken with another set of windows. The resulting overlap of the data sets is good; however, we estimate a common systematic uncertainty of 5\%-10\% for the target thickness of the three individual data sets and the corresponding cross section values.

The results of our measurements are displayed in Fig. 1 together with the cross sections calculated with the YK model [5] and the HS model [3], respectively. Both models are equivalent over the displayed energy region. For the gas scattering results, the agreement is remarkable. It corroborates the quantum-mechanical basis for neutron- ${ }^{2} \mathrm{H}_{2}$ scattering in the center of mass (c.m.) system as well as the averaging over the Maxwell-Boltzmann spectrum for the ${ }^{2} \mathrm{H}_{2}$ gas molecules. In contrast to YK, HS make the thermal averaging at the last stage of the calculation. At low energies the cross section tends to a $1 / \sqrt{E}$ behavior, which arises from the folding of the roughly constant scattering cross section in the c.m. system at very low energies [3] with a Maxwell-Boltzmann distribution [see Eq. (57) in [3] ] and formally finding the limit as $E$ tends to zero [32], leading to

$$
\sigma_{E \rightarrow 0}^{\mathrm{HS}}(E)=\frac{\bar{\sigma}_{0 \leftarrow 0}^{\mathrm{HS}}}{\sqrt{E}} \sqrt{\frac{k_{B} T}{\pi}},
$$

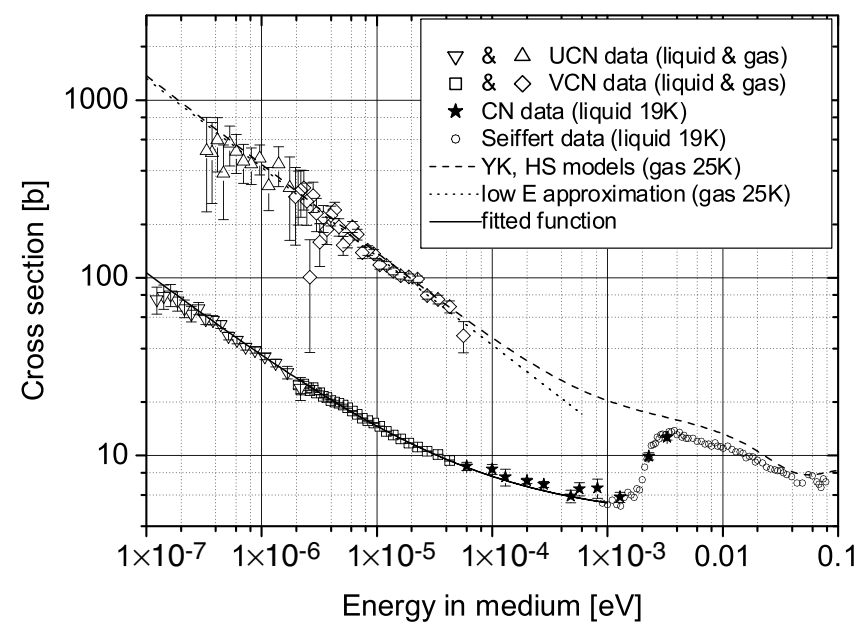

FIG. 1. Measured total neutron scattering cross sections per molecule as a function of the in-medium neutron energy for gaseous (upper curve) and liquid ${ }^{2} \mathrm{H}_{2}$ (lower curve) at 25 and $19 \mathrm{~K}$, respectively. The data of Seiffert [9] for cold neutrons on liquid ${ }^{2} \mathrm{H}_{2}$ are also included. The Hamermesh and Schwinger [3] and the Young and Koppel [5] models reproduce the measured cross sections of ${ }^{2} \mathrm{H}_{2}$ in the gas phase. Liquid ${ }^{2} \mathrm{H}_{2}$ below $1 \mathrm{meV}$ is described with an empirical $1 / \sqrt{E^{\prime}}$ function (see text).

with Boltzmann's constant $k_{B}$, temperature $T$, and the elastic ground state cross section $\left(J^{\prime}=0 \leftarrow J=0\right) \bar{\sigma}_{0 \leftarrow 0}^{\mathrm{HS}}$ which is obtained from averaging the cross section $\sigma_{0 \leftarrow 0}^{\mathrm{HS}}(E)$ [see Eqs. (52) and (53) in [3] ] over a MaxwellBoltzmann distribution of the ${ }^{2} \mathrm{H}_{2}$ molecules. In particular, $\sigma_{0 \leftarrow 0}^{\mathrm{HS}}(0)=17.5 \mathrm{~b}$, and using $T=25 \mathrm{~K}$,

$$
\bar{\sigma}_{0 \leftarrow 0}^{\mathrm{HS}}=16.0 \mathrm{~b} \text {. }
$$

Equation (2) may be obtained in a slightly more general and illuminating way: The cross section in Eq. (1) is an average over a range of c.m. system velocities coming from the (singular) incident velocity, $v$, and the velocity spectrum, $f\left(v_{M}\right)$, of the molecules. If we consider the reaction rate for neutrons with an incident velocity negligibly small compared to that of the gas, then we can write

$$
\rho \sigma(v) d=\int_{0}^{\infty} \rho \bar{\sigma}_{0 \leftarrow 0} v_{M} \frac{d}{v} f\left(v_{M}\right) d v_{M}=\rho \bar{\sigma}_{0 \leftarrow 0} \frac{\bar{v}_{M}}{v} d .
$$

Substituting the value for the average velocity of gas for a Maxwell-Boltzmann distribution, $\bar{v}_{M}=\sqrt{8 k_{B} T /(\pi M)}$, one obtains Eq. (2) with $\bar{\sigma}_{0 \leftarrow 0}^{\mathrm{HS}}=\bar{\sigma}_{0 \leftarrow 0}$.

In the following we extract the cross section $\bar{\sigma}_{0 \leftarrow 0}$ from the data: The measured cross section values can be fitted to a relationship of the form

$$
\sigma=\sigma_{0}+\frac{\alpha}{\sqrt{E^{\prime}}}
$$

and if we fix $\sigma_{0}$ to the incoherent value of $4.1 \mathrm{~b}$ [33], then we find that the ${ }^{2} \mathrm{H}_{2}$ gas values require $\alpha_{\text {gas }}=(0.40 \pm$ $0.02) \mathrm{b} \cdot \sqrt{\mathrm{eV}}$. For sufficiently small energies one gets $\alpha=$ $\bar{\sigma}_{0 \leftarrow 0} \sqrt{k_{B} T / \pi}$. Inserting the measurement temperature of 
$25 \mathrm{~K}$ yields a value of $\bar{\sigma}_{0 \leftarrow 0}$ of $(15.5 \pm 0.8) \mathrm{b}$ in excellent agreement with the value for $\bar{\sigma}_{0 \leftarrow 0}^{H S}$ [see Eq. (3)].

The liquid ${ }^{2} \mathrm{H}_{2}$ cross sections obtained in this experiment confirm the results of Seiffert [9]. They show also that the gas models $[3,5]$ do not describe the results for liquid ${ }^{2} \mathrm{H}_{2}$, particularly in the region between 1 to $7 \mathrm{meV}$. However, the results below $1 \mathrm{meV}$ can be fitted to the form of Eq. (5) and yield a value for $\sigma_{0}$ of (4.4 \pm $0.1) \mathrm{b} /$ molecule and $\alpha_{\text {liq }}=(32.4 \pm 0.2) \mathrm{mb} \cdot \sqrt{\mathrm{eV}}$. The value for $\sigma_{0}$ is in reasonable agreement with the accepted value for the incoherent cross section [33]. The value for $\alpha_{\text {liq }}$ together with the value of $\bar{\sigma}_{0 \leftarrow 0}$ obtained from the gas results can be used in Eq. (4) to extract an average velocity of about $(28 \pm 2) \mathrm{m} / \mathrm{s}$. Surprisingly, one finds almost the same value for the ${ }^{2} \mathrm{H}_{2}$ transport velocity from deuterium self-diffusion, using (see, e.g., Ref. [34])

$$
D_{44}=\frac{1}{3} \bar{v}_{M} \lambda_{t} \text {. }
$$

The diffusion coefficient for liquid ${ }^{2} \mathrm{H}_{2}$ at $19 \mathrm{~K}$ is $D_{44}=$ $3.3 \times 10^{-9} \mathrm{~m}^{2} / \mathrm{s}$ [31], the transport free path is of the order of the intermolecular separation, $\lambda_{t} \approx 4 \times 10^{-10} \mathrm{~m}$, and thus $\bar{v}_{M} \approx 25 \mathrm{~m} / \mathrm{s}$. This result suggests that the HS model may actually apply also to liquid ${ }^{2} \mathrm{H}_{2}$ at low energies when the proper velocity distribution is used. The region between 1 and $7 \mathrm{meV}$ can then be understood to be entirely due to the onset of coherent scattering (compare, e.g., Refs. [9,35]).

In conclusion, we have presented a low-energy extension to available neutron cross section data for gaseous and liquid ${ }^{2} \mathrm{H}_{2}$. The agreement of the HS [3] and YK [5] models and the gaseous data is excellent. The liquid data at energies below 4 to $7 \mathrm{meV}$ are not described by these gas models. It is, however, possible to fit the liquid data at low energies with a simple $1 / \sqrt{E^{\prime}}$ dependence and to give a description of the liquid cross sections using the same energy-independent c.m. system cross section as for ${ }^{2} \mathrm{H}_{2}$ gas (and in agreement with HS) and an average molecular velocity in the liquid very close to the one expected from deuterium diffusion. It will be interesting to see how the new data can be accommodated by more sophisticated liquid models.

This work was performed at the Swiss Spallation Neutron Source SINQ, Paul Scherrer Institut, Villigen, Switzerland, and at the Institute Laue-Langevin (ILL), Grenoble, France. We are grateful to various technical support services, whose outstanding efforts have made these experiments possible. We especially acknowledge the help of W. Arrigoni, T. Brenner, K. Kohlik, and P. Schurter. This work is supported by the Swiss National Science Foundation, Grant No. 2100-067840.

*Also at Institute for Technical Chemistry, ETH Zürich, Switzerland.

${ }^{\dagger}$ Also at Physik-Institut, University of Zürich, Switzerland.
ॠCorresponding author.

Electronic address: klaus.kirch@psi.ch

${ }^{\S}$ Electronic address: http://ucn.web.psi.ch

"Also at Paul Scherrer Institut, CH-5232 Villigen PSI, Switzerland.

IIAlso at Research Center for Nuclear Physics, Osaka, Japan.

[1] J. Schwinger and E. Teller, Phys. Rev. 52, 286 (1937).

[2] M. Hamermesh and J. Schwinger, Phys. Rev. 55, 671 (1939); see p. 679.

[3] M. Hamermesh and J. Schwinger, Phys. Rev. 69, 145 (1946).

[4] A. C. Zemach and R. J. Glauber, Phys. Rev. 101, 118 (1956).

[5] J. A. Young and J. U. Koppel, Phys. Rev. 135, A603 (1964).

[6] J. U. Koppel and J. A. Young, Nukleonika 8, 40 (1966).

[7] G. L. Squires and A. T. Stewart, Proc. R. Soc. A 230, 19 (1955).

[8] W. Schott, Z. Phys. 231, 243 (1970).

[9] W.-D. Seiffert, Report No. EUR 4455 d, 1970.

[10] M. Utsuro, Z. Phys. B 27, 111 (1977).

[11] J. Keinert and J. Sax, Kerntechnik 51, 19 (1987).

[12] W. Bernnat et al., Physica (Amsterdam) 234-236B, 1199 (1997).

[13] J. R. Granada et al., J. Neutron Res. 11, 25 (2003).

[14] N. Morishima, Ann. Nucl. Energy 27, 505 (2000).

[15] N. Morishima and Y. Nishikawa, Ann. Nucl. Energy 31, 737 (2004).

[16] http://ucn.web.psi.ch/.

[17] F. Atchison et al., Phys. Rev. C 71, 054601 (2005).

[18] A. Serebrov et al., Nucl. Instrum. Methods Phys. Res., Sect. A 440, 658 (2000).

[19] C. L. Morris et al., Phys. Rev. Lett. 89, 272501 (2002).

[20] A. Saunders et al., Phys. Lett. B 593, 55 (2004).

[21] In fact, we are not aware of any cross section data for deuterium below a neutron energy of $0.9 \mathrm{meV}$, the lowest energy of [9].

[22] J. Kohlbrecher and W. Wagner, J. Appl. Crystallogr. 33, 804 (2000).

[23] A. Steyerl et al., Phys. Lett. A 116, 347 (1986).

[24] V. K. Ignatovich, Ultracold Neutrons (Clarendon Press, Oxford, 1990).

[25] $U=\frac{2 \pi \hbar^{2}}{m_{n}} N_{0} b_{\text {coh }}$ was calculated using for the liquid ${ }^{2} \mathrm{H}_{2}$ : $N_{0}=\stackrel{m_{n}}{0} 042 \mathrm{~mol} / \mathrm{cm}^{3}$ [31] and $b_{\text {coh }}=2 \times 6.67 \mathrm{fm} \mathrm{[33].}$

[26] M. Kasprzak, Diploma thesis, Jagiellonian University, Krakow, 2004, nucl-ex/0407022.

[27] F. Atchison et al. (to be published).

[28] K. Bodek et al., Nucl. Instrum. Methods Phys. Res., Sect. A 533, 491 (2004).

[29] C.-Y. Liu, A. R. Young, and S. K. Lamoreaux, Phys. Rev. B 62, R3581 (2000).

[30] K. G. McLennan and E. MacA Gray, Meas. Sci. Technol. 15, 211 (2004).

[31] P.C. Souers, Hydrogen Properties for Fusion Energy (University of California, Berkeley, 1986).

[32] Details about the derivation of the limit of slow neutron velocities compared to the molecular velocities are found, e.g., in Ref. [4], Eq. (5.4) and p. 127.

[33] V.F. Sears, Neutron News 3, 26 (1992).

[34] J.M. Pendlebury, Kinetic Theory (Adam Hilger Ltd., Bristol and Boston, 1985).

[35] S. J. Talhouk et al., J. Chem. Phys. 48, 1273 (1968). 